\title{
Combination use of triamcinolone acetonide and immunotherapy as a new therapeutic option in alopecia totalis
}

\author{
Takashi Yoshimasu ${ }^{1,2^{*}}$, Naoya Mikita ${ }^{1}$, Takaharu Ikeda ${ }^{1}$, Nobuo Kanazawa ${ }^{1}$, Fukumi Furukawa ${ }^{1,3}$, \\ Masatoshi Jinnin ${ }^{1}$ \\ ${ }^{1}$ Department of Dermatology, Wakayama Medical University, Japan \\ ${ }^{2}$ Department of Dermatology, Arida Municipal Hospital, Japan \\ ${ }^{3}$ Takatsuki Red Cross Hospital, Osaka, Japan
}

\begin{abstract}
Alopecia totalis (AT) with body hair loss is the most severe type of alopecia areata (AA). The ability to develop hair is suggested to be poor in such severe AA, because AT does not respond to corticosteroid pulses and immunotherapy using squaric acid dibutylester (SADBE) or diphenylcyclopropenone (DPCP). The purpose of this study is to assess the possibility of hair regrowth in AT with body hair loss. Ten patients with AT who did not respond to topical immunotherapies, received triamcinolone acetonide (TA) injections. Undiluted or 2-fold diluted solutions of TA were prepared and $0.1-0.2 \mathrm{~mL}$ of either of the two solutions was administered to each patient. In total, $2 \mathrm{~mL}$ of the selected solution was injected monthly into each area. In cases where vellus hair developed after the injections, we restarted the immunotherapy using SADBE or DPCP and continued the therapies for more than half a year. The development of vellus hair after TA injections was defined as a good response. Complete response rate to the topical injection of TA was $10 \%(1 / 10)$, however the partially good response rate was $60 \%(6 / 10)$. The good responders showed the anagen stage of hair follicle after TA injections. Furthermore, the complete responder to TA showed susceptibility to the subsequent immunotherapy and more regrowth of hair was seen. Even if patients with AT have suffered for a prolonged period since onset, it is possible to recover the hair cycle if they show susceptibility to intralesional corticosteroid and subsequent immunotherapy.
\end{abstract}

Keywords: alopecia totalis; triamcinolone acetonide; SADBE; DPCP; immunotherapy

\section{ARTICLE INFO}

Received: March 15, 2020

Accepted: April 11, 2020

Available online: April 21, 2020

*CORRESPONDING AUTHOR Takashi Yoshimasu, 811-1, Kimiidera, Wakayama City, Wakayama, 6410012, Japan; yosshii8@jg8.so-net.ne.jp

\section{CITATION}

Yoshimasu T, Mikita N, Ikeda T, et al. Combination use of triamcinolone acetonide and immunotherapy as a new therapeutic option in alopecia totalis Trends Immunother 2020; 4(1): 1-4. doi: 10.24294/ti.v4.i1.149.

\section{COPYRIGHT}

Copyright (C) 2020 by author(s) and EnPress Publisher LLC. This work is licensed under the Creative Commons Attribution-NonCommercial 4.0 International License (CC BY-NC 4.0). http://creativecommons.org/licenses/ by/4.0/

\section{Introduction}

Alopecia areata (AA) is usually characterized by limited alopecic patches on the scalp. However, more severe forms may affect the entire scalp (alopecia totalis: AT) or body hair including eyebrows, cilia, axillary hair and hair of the pubis (alopecia universalis: AU). The possibility of hair regrowth is suggested to be poor in such AT with body hair loss, because AT does not respond to steroid pulses and immunotherapy using squaric acid dibutylester (SADBE) or diphenylcyclopropenone (DPCP) ${ }^{[1]}$. Intralesional corticosteroids are not as effective for the treatment of AT as they are for localized alopecia ${ }^{[2]}$. However, the recommended degree of intralesional corticosteroid is higher than other therapies in alopecia areata with up to $25 \%$ hair $\operatorname{loss}^{[3]}$. It is supposed that the susceptibility of AT patients to intralesional corticosteroid is diverse. The purpose of this study is to assess the susceptibilities to intralesional corticosteroid and subsequent immunotherapy using SADBE or DPCP in AT patients with body hair loss.

\section{Materials and methods}

Ten patients with AT (male: 1, female: 9; aged 12-43 years) were enrolled in this study between 2008 and 2017. All of them had either partial or complete body hair loss. Topical immunotherapy using SADBE or DPCP for the AT patients was approved by the ethics committee of Wakayama Medical University. Informed consent was obtained from each patient. To exclude AA associated with other diseases, biological examinations were carried out including tests for serum zinc, serum iron, HBs antigen, and hepatitis $\mathrm{C}$ 
antibody, a serologic test for syphilis and Treponema pallidum hemagglutination, and a thyroid function test and a measurement of anti nuclear antibody.

We started a modified topical immunotherapy using SADBE or DPCP starting with $0.01 \%$ for the patients with AT before starting triamcinolone acetonide (TA) injections ${ }^{[1]}$. The immunotherapy was continued at optimal concentration in each patient for several months. The optimal concentration was decided in each patient to had seen continuous erythema for 4 to 5 days after the topical immunotherapy. Undiluted or 2-fold diluted solution of TA was prepared and $0.1-0.2 \mathrm{~mL}$ of the either solution was injected into each area. The choice of solution was determined by the patient's reaction to the initial injection of a double-diluted solution. Those who showed no adverse side effects subsequently received the undiluted solution. In total, $2 \mathrm{~mL}$ of the solution was injected monthly at least several times into the AT lesion in each patient. Repeated TA injections at the same site were avoided to prevent skin atrophy. Susceptibility to TA was assessed by whether vellus hair had developed in the patients with AT. Irrespective of the development of vellus hair in the patients with AT after several TA injections, we restarted the immunotherapy using SADBE or DPCP and continued the therapy every two weeks for more than half a year. We assessed clinical changes over time. Skin biopsies were performed before or after the intralesional corticosteroid in some patients.

\section{Results}

In this study, the AT patients with body hair loss had an average duration of more than 5 years from the onset of alopecia (Table 1). No one showed susceptibility to the immunotherapy using SADBE or DPCP before starting TA injections. Complete response rate to the topical injections of TA was $10 \%(1 / 10)$ (Figure 1a. b, Table 1) however the partially good response rate was 60\% (6/10) (Table 1). The good responder showed the anagen stage of hair follicle after TA injection (Figure 2A) and trichoscopic examination showed the development of vellus hair without exclamation mark hair (Figure 2B). Furthermore, she showed susceptibility to the subsequent immunotherapy and more regrowth of hair was seen up to 10 months after starting TA injections (Figure 1C-F). Some of the AT patients were partially susceptible to TA injections (Figure $3 \mathrm{a} . \mathrm{b})$ and hair follicles in the anagen stage were seen (Figure 3C). Some of the partial responders to TA injections were also partially susceptible to the immunotherapy of SADBE or DPCP (Table 1), however such patients did not show complete hair regrowth satisfactorily. There were no obvious side effects to the discontinuation of the therapies using TA injections and the immunotherapy during the study.
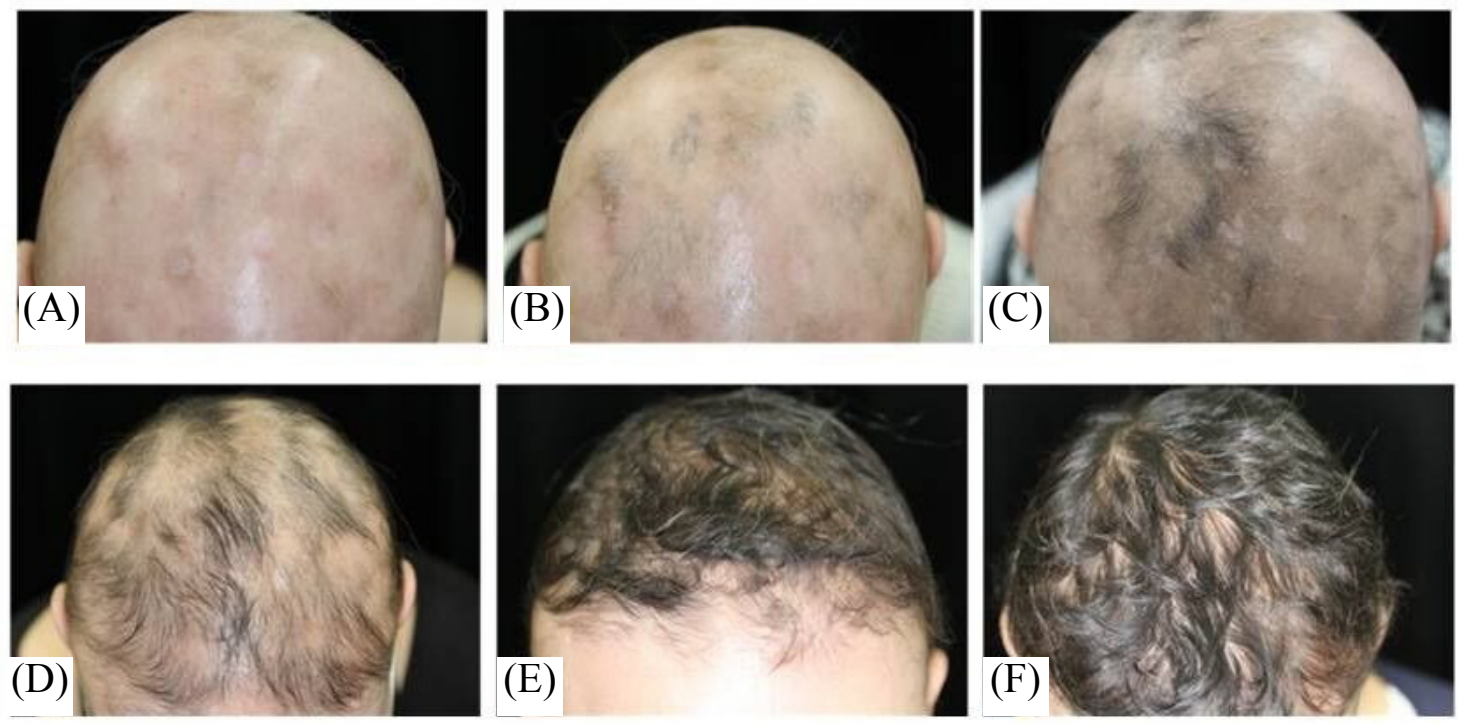

Figure 1. Clinical changes in alopecia totalis with body hair loss susceptible to intralesional triamcinolone acetonide (TA) and subsequent immunotherapy (Patient No. 1). (A) Alopecia totalis before starting intralesional TA injections. (B)Vellus hair was developed after monthly TA injections. (C) Immunotherapy using SADBE showed hair regrowth following TA injections. (D-F) Hair regrowth was accelerated by immunotherapy every two weeks from 6 months to 10 months after the TA injections. 

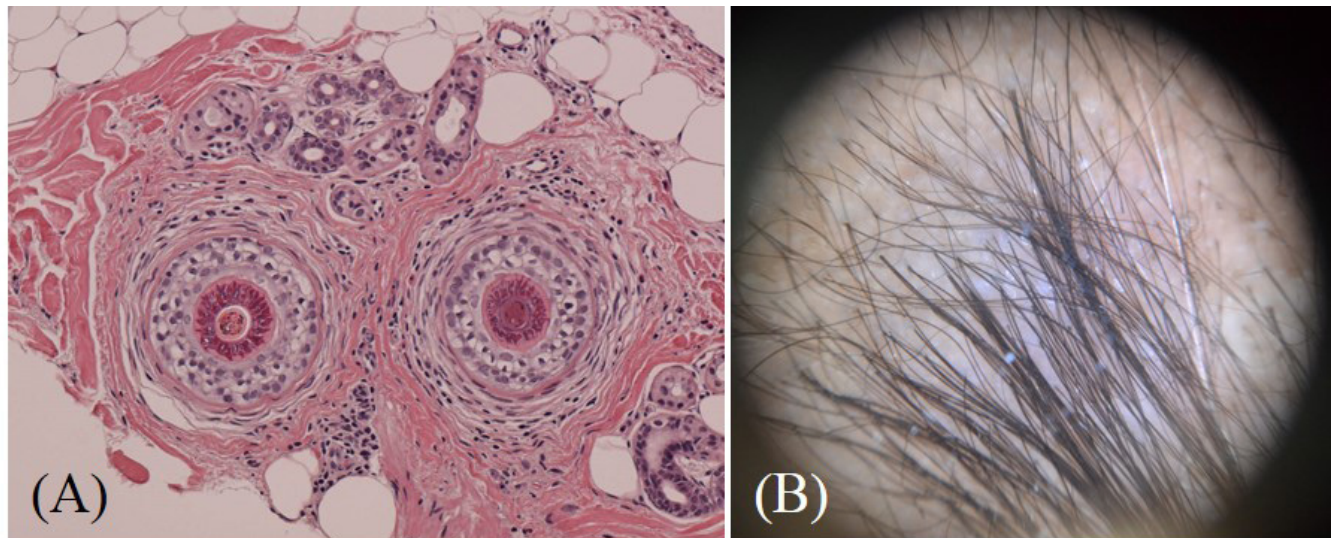

Figure 2. Histopathological finding and trichoscopic findings after the development of vellus hair. (Patient No. 1). (A) Hair follicles in the anagen stage were seen after two injections of half-diluted solution of TA (HE stain, $\times 200$ ). (B) Trichoscopic finding showed the development of vellus hair without exclamation mark hair.
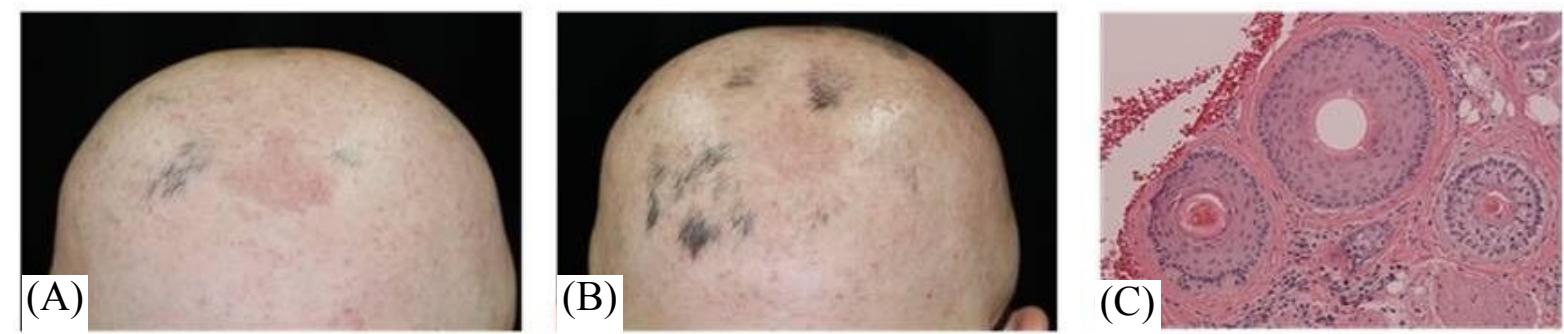

Figure 3. Clinical findings in AT with body hair loss partially susceptible to TA but not immunotherapy (Patient No. 7). (A) Alopecia totalis before starting an intralesional TA injection. (B) Intralesional injections of undiluted TA induced partial development of vellus hair. (C) Anagen hair follicles were seen after four times injections of undiluted solution of TA (HE stain, $\times 200)$.

Table 1. Summary of susceptibilities to both TA and immunotherapy in AT patients.

\begin{tabular}{|c|c|c|c|c|c|c|c|c|c|}
\hline $\begin{array}{l}\text { Pt } \\
\text { No. }\end{array}$ & Age & Sex & Severity & $\begin{array}{c}\text { Duration } \\
\text { of } \\
\text { alopecia } \\
\text { (years) }\end{array}$ & $\begin{array}{l}\text { Immuno- } \\
\text { therapy }\end{array}$ & $\begin{array}{l}\text { Susceptibility } \\
\text { to immuno- } \\
\text { therapy } \\
\text { before TA } \\
\text { injection }\end{array}$ & $\begin{array}{c}\text { Susceptibility } \\
\text { to TA injection } \\
\text { (Vellus hair } \\
\text { development) }\end{array}$ & $\begin{array}{l}\text { Susceptibility } \\
\text { to immuno- } \\
\text { therapy after } \\
\text { TA injection }\end{array}$ & $\begin{array}{c}\text { Hair } \\
\text { regrowth }\end{array}$ \\
\hline 1 & 41 & $\mathrm{~F}$ & S5B2 & 3 & SADBE & - & + & + & + \\
\hline 2 & 28 & $\mathrm{~F}$ & S5B2 & 5 & SADBE & - & $+/-$ & $+/-$ & $+/-$ \\
\hline 3 & 18 & $\mathrm{~F}$ & S5B1 & 10 & $\begin{array}{l}\text { SADBE, } \\
\text { DPCP }\end{array}$ & - & $+/-$ & $+/-$ & $+/-$ \\
\hline 4 & 20 & $\mathrm{~F}$ & S5B2 & 3 & DPCP & - & $+/-$ & $+/-$ & $+/-$ \\
\hline 5 & 43 & $\mathrm{~F}$ & S5B1 & 3 & DPCP & - & $+/-$ & $+/-$ & $+/-$ \\
\hline 6 & 28 & $\mathrm{~F}$ & S5B1 & 4 & DPCP & n.d. & $+/-$ & - & - \\
\hline 7 & 37 & M & S5B1 & 1 & SADBE & - & $+/-$ & - & - \\
\hline 8 & 42 & F & S5B2 & 9 & SADBE & - & - & - & - \\
\hline 9 & 26 & $\mathrm{~F}$ & S5B2 & 10 & DPCP & - & - & - & - \\
\hline 10 & 12 & $\mathrm{~F}$ & S5B2 & 6 & n.d. & n.d. & - & n.d. & - \\
\hline
\end{tabular}

(S5: 100\% hair loss, B1: some body hair loss, B2: 100\% body hair loss) (TA: triamcinolone acetonide) (Susceptibility; +: susceptible, +/-: partially susceptible, -; negative) (Hair regrowth; +: complete hair growth, +/-: partial hair growth, -: no hair growth) 


\section{Discussion}

Various treatments are known in AT and alopecia universalis (AU), and although however certain treatments have shown significant hair regrowth, no treatment is completely effective $\mathrm{e}^{[4]}$. Recently, the efficacy of hydroxychloroquine ${ }^{[5,6]}$ in the treatment of AT has been reported ${ }^{[7]}$ but its use for extensive alopecia areata is still controversial ${ }^{[8]}$. Tofatinib is also reported to show efficacy in severe AA including $\mathrm{AT}$ and $\mathrm{AU}^{[9]}$.

In this study, the patients with AT had an average duration of more than 5 years from the onset of alopecia. Patients with AT for more than 6 months from the onset are resistant to steroid pulses and immunotherapy using SADBE or DPCP ${ }^{[1,10]}$. It is well known that the method using TA is most suitable for treating patchy hair loss of a limited extent. Despite this, because of the variability of individual response, we decided to try TA injections on the patients with AT. Susceptibility to TA is supposed to be diverse in AA including $\mathrm{AT}$ and $\mathrm{AU}$, however intralesional corticosteroid is recommended in AA with up to $25 \%$ hair $\operatorname{loss}^{[3]}$. One AT patient showed susceptibility to TA injections and complete vellus hair was seen in our study. Hair follicles in the anagen stage were seen histologically. The patient also showed susceptibility to the subsequent immunotherapy using SADBE, however she did not respond to it before the TA injections. Adequate vellus hair may be essential to in order to be susceptible to the subsequent immunotherapy. On the other hand, it is also possible that the susceptibility to immunotherapy sustains the anagen stage of the hair cycle. Even if patients with AT have suffered for a prolonged period since onset, it is possible to recover the hair cycle if they show susceptibility to intralesional corticosteroid and subsequent immunotherapy. The method using TA and subsequent immunotherapy for AT patients with body hair loss showed only a $10 \%$ success rate. However, as long as AT patients have a possibility to recover their hair, it is necessary to try various methods to recover the hair cycle even though the method has a low success rate.

\section{Conflict of interest}

This work was supported in part by a grant from Mitsubishi Tanabe Pharma to TY and a grant from Novartis Pharmaceuticals Corp. to NM.

\section{References}

1. Yoshimasu T, Furukawa F. Modified immunotherapy for alopecia areata. Autoimmun Rev 2016; 15(7): 664-667. doi: 10.1016/j.autrev.2016.02.021.

2. Messenger AG, McKillop J, Farrant P, et al. British Association of Dermatologists' guidelines for the management of alopecia areata 2012 . Br J Dermatol 2012; 166(5): 916-926. doi: 10.1111/ j.1365-2133.2012.10955.x

3. Arase S, Tsuboi R, Yamazaki M, et al. The Japanese Dermatological Association's guidelines for the management of alopecia areata 2010. Jpn J Dermatol 2010; 120: 1841-1859. doi: 10.14924/ dermatol.120.1841.

4. Kassira S, Korta DZ, Chapman LW, et al. Review of treatment for alopecia totalis and alopecia universalis. Int J Dermatol 2017; 56(8): 801-810. doi: 10.1111/ijd.13612.

5. Furukawa F. Hydroxychloroquine in lupus erythematosus, a new horizon of the old drug. Trends Immunother 2017; 1(3): 99-100. doi: 10.24294/ti.v1.i3.127.

6. Hirakawa Y, Okuno A, Kimura D, et al. Hydroxychloroquine enhanced urticarial reaction in a patient with discoid lupus erythematosus. Trends Immunother 2017; 1(3): 121-123. doi: 10.24294/ ti.v1.i3.125.

7. Stephan F, Habre M, Tomb R. Successful treatment of alopecia totalis with hydroxychloroquine: Report of 2 cases. J AM Acad Dermatol 2013; 68(6): 1048-1049. doi: 10.1016/j.jaad.2013.02.011.

8. Nissen CV, MD, Wulf HC. Hydroxychloroquine is ineffective in treatment of alopecia totalis and extensive alopecia areata: A case series of 8 patients. JAAD Case Rep 2016; 2(2): 117-118. doi: 10.1016/j.jdcr.2016.01.005

9. Liu LY, Craiglow BG, Dai F, et al. Tofacitinib for the treatment of severe alopecia areata and variants: A study of 90 patients. J Am Acad Dermatol 2017; 76(1): 22-28. doi: 10.1016/j.jaad.2016.09.007.

10. Yoshimasu T, Kanazawa N, Yamamoto Y, et al. Multiple courses of pulse corticosteroid therapy for alopecia areata. J Dermatol 2016; 43(9): 10751077. doi: 10.1111/1346-8138.13388. 